\title{
Near-to-Final MINOS Oscillation Results
}

Jeffrey K. de Jong for the MINOS Collaboration

${ }^{*}$ Department of Physics, University of Oxford, Oxford, OX1 3RH, United Kingdom

\begin{abstract}
The MINOS (Main Injector Neutrino Oscillation Search) experiment has been collecting NuMI beam neutrino data since 2005 and atmospheric neutrino data since 2003. The NuMI beam delivered its last Proton on Target (POT) for MINOS neutrino production in April 2012 prior to its shutdown and upgrade for NOvA. This paper presents MINOS' near-to-final measurements of the neutrino oscillation parameters obtained when using the complete MINOS data set.
\end{abstract}

Keywords: MINOS, NuMI, beam neutrinos, atmospheric neutrinos

\section{The MINOS experiment}

MINOS [1] is a two detector long-baseline neutrino oscillation experiment. The 0.98 kton near detector is located approximately $1 \mathrm{~km}$ from the NuMI target. The $5.4 \mathrm{kton}$ far detector is located $734 \mathrm{~km}$ further downstream and $705 \mathrm{~m}$ underground (2070 meters of water equivalent) in the Soudan Underground Laboratory in northern Minnesota. The two detectors are functionally identical, to minimize beam and cross-section uncertainties, and consist of alternating steel and scintillating planes. The scintillating planes are comprised of strips measuring $4.1 \mathrm{~cm}$ wide and $1.0 \mathrm{~cm}$ thick. Each scintillating plane is attached to a $2.54 \mathrm{~cm}$ thick toroidally magnetized $(1.3 \mathrm{~T})$ steel plane. Neighboring scintillating layers are rotated by $90^{\circ}$ to allow for three dimensional track reconstruction. The magnetic field permits charge-sign (and momentum) determination of the muons produced in charged current (CC) interactions allowing MINOS to discriminate between $v_{\mu}$ and $\bar{v}_{\mu}$ on an event-by-event basis.

The NuMI neutrino beam [2] is generated by the decay of focused $\pi \mathrm{s}$ and Ks produced when $120 \mathrm{GeV}$ protons strike the NuMI target. The NuMI neutrino beam operated between March 2005 and April 2012. The

Email address: jeffrey.dejong@physics.ox.ac.uk (Jeffrey K. de Jong for the MINOS Collaboration) neutrino energy can be controlled by the location of the target with respect to the focusing horns. Changing the polarity of the focusing horns allows MINOS to either produce a predominantly muon neutrino beam, or a beam with a larger anti-neutrino component. In the standard configuration, the Low Energy (LE) Forward Horn Current (FHC, $v_{\mu}$-dominated) mode of operation the beam is comprised of $91.7 \% v_{\mu}, 7.0 \% \bar{v}_{\mu}$ and $1.3 \%\left(v_{e}+\bar{v}_{e}\right)$ and peaks around a few GeV. With the horn polarity reversed (RHC, $\bar{v}_{\mu}$-enhanced mode) the beam is $39.9 \% v_{\mu}, 58.1 \% \bar{v}_{\mu}$ and $2.0 \%\left(v_{e}+\bar{v}_{e}\right)$. A total of $10.71 \times 10^{20}$ POTs were collected in the $v_{\mu}$-dominated mode and $3.36 \times 10^{20}$ POTs in the $\bar{v}_{\mu}$-enhanced mode.

The far detector has been collecting atmospheric neutrino data since August 2003 [3]. To reduce backgrounds for the atmospheric neutrino analysis a scintillator-based veto shield was installed above the far detector and is used to tag cosmic ray muons entering the detector. Restricting data to periods where both the far detector and veto shield were fully operational results in 2553 live-days corresponding to an exposure of 37.88 kton-yrs.

\section{Disappearance Analyses}

In the simple two-flavour approximation, the survival probability of a $v_{\mu}\left(\right.$ or $\left.\bar{v}_{\mu}\right)$ with an energy $\mathrm{E}[\mathrm{GeV}]$ trav- 

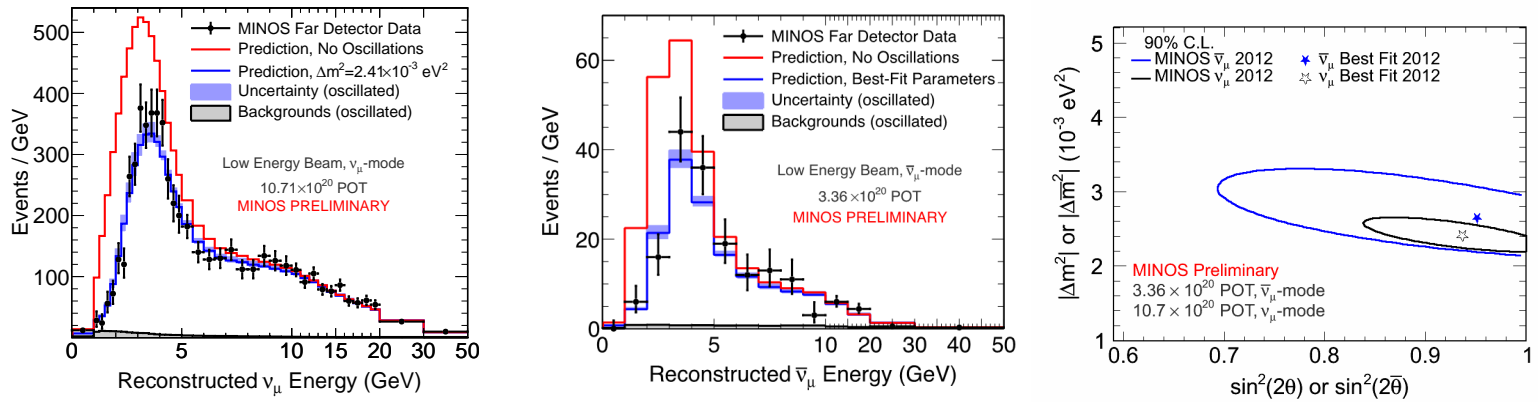

Figure 1: The reconstructed energy spectrum of selected $v_{\mu}$ (left) and $\overline{v_{\mu}}$ (middle) at the MINOS far detector. The red line is the prediction in the absence of oscillations and the blue line is the best fit to the data. (right) The $90 \%$ C.L. contours are shown on the right for the $v_{\mu}$ (black) and $\bar{v}_{\mu}$ (blue) data sets when they are fit separately. The best fit oscillation parameters are in good agreement.
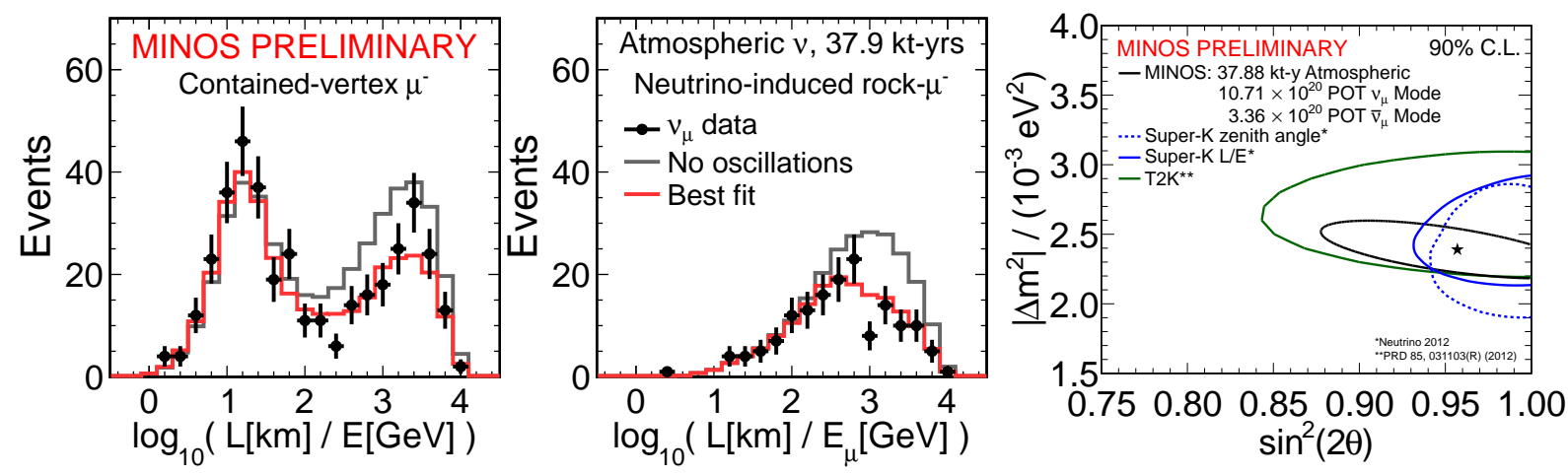

Figure 2: $\log _{10}(L / E)$ distributions for contained-vertex neutrino interactions (left) and neutrino-induced rock muon events (middle). The black lines are the predictions assuming no oscillations. The red lines indicate the best fit to all the MINOS data assuming that neutrinos and anti-neutrinos oscillate with the same parameters. The $90 \%$ C.L. contour for the fit to all (beam and atmospherics) the MINOS data is shown in black on the right and is compared to the recent Super-K and T2K results.

eling a distance $\mathrm{L}[\mathrm{km}]$ is given by:

$$
P\left(v_{\mu} \rightarrow v_{\mu}\right)=1-\sin ^{2}(2 \theta) \sin ^{2}\left(\frac{1.27 \Delta m^{2} L}{E}\right)
$$

where $\Delta m^{2}$ is the mass splitting and $\theta$ is the mixing angle. The $v_{\mu} / \bar{v}_{\mu}$ disappearance analyses are performed using charged current (CC) interactions, $v_{\mu}\left(\bar{v}_{\mu}\right)+N \rightarrow$ $\mu^{-}\left(\mu^{+}\right)+X$. These interactions are characterized by a hadronic shower at the event vertex (if the vertex is in the detector) and a long (anti-)muon track.

For the beam analyses $[4,6]$ the far detector spectrum is predicted by tuning the Monte-Carlo to reproduce the measured near detector neutrino spectrum and then extrapolating that to the far detector. Figure 1 plots the reconstructed neutrino and anti-neutrino spectrum at the far detector. The neutrino energy is determined by summing the hadronic and leptonic energy of the event. In total we expect 3564 (312) $v_{\mu}\left(\bar{v}_{\mu}\right)$ CC events in the far detector and observe 2894 (226). When fit separately we measure $\sin ^{2}(2 \theta)=0.94_{-0.05}^{+0.04}$ and $\left|\Delta m^{2}\right|=$ $2.41_{-0.10}^{+0.11} \times 10^{-3} \mathrm{eV}^{2}$ for the neutrino data, and $\sin ^{2}(2 \theta)>$ $0.78(90 \%$ C.L. $)$ and $\left|\Delta m^{2}\right|=2.64_{-0.27}^{+0.28} \times 10^{-3} \mathrm{eV}^{2}$ for the anti-neutrino data.

For the atmospheric neutrino analysis the neutrino spectrum is determined using the Bartol 3D neutrino flux model [7]. The atmospheric $v_{\mu} \bar{v}_{\mu}$ samples [3] are separated into contained vertex events, where the interaction vertex is inside the fiducial volume of the detector, and neutrino-induced muons, where the vertex is outside the detector but the muon enters the detector. In the first class of events the neutrino energy is reconstructed as usual. In the latter the analysis is performed on the reconstructed muon energy. Figure 2 plots the distribution of $L / E$ for the two classes of neutrino interactions as well as the confidence limit contour obtained when both the beam and atmospheric data sets are fit 

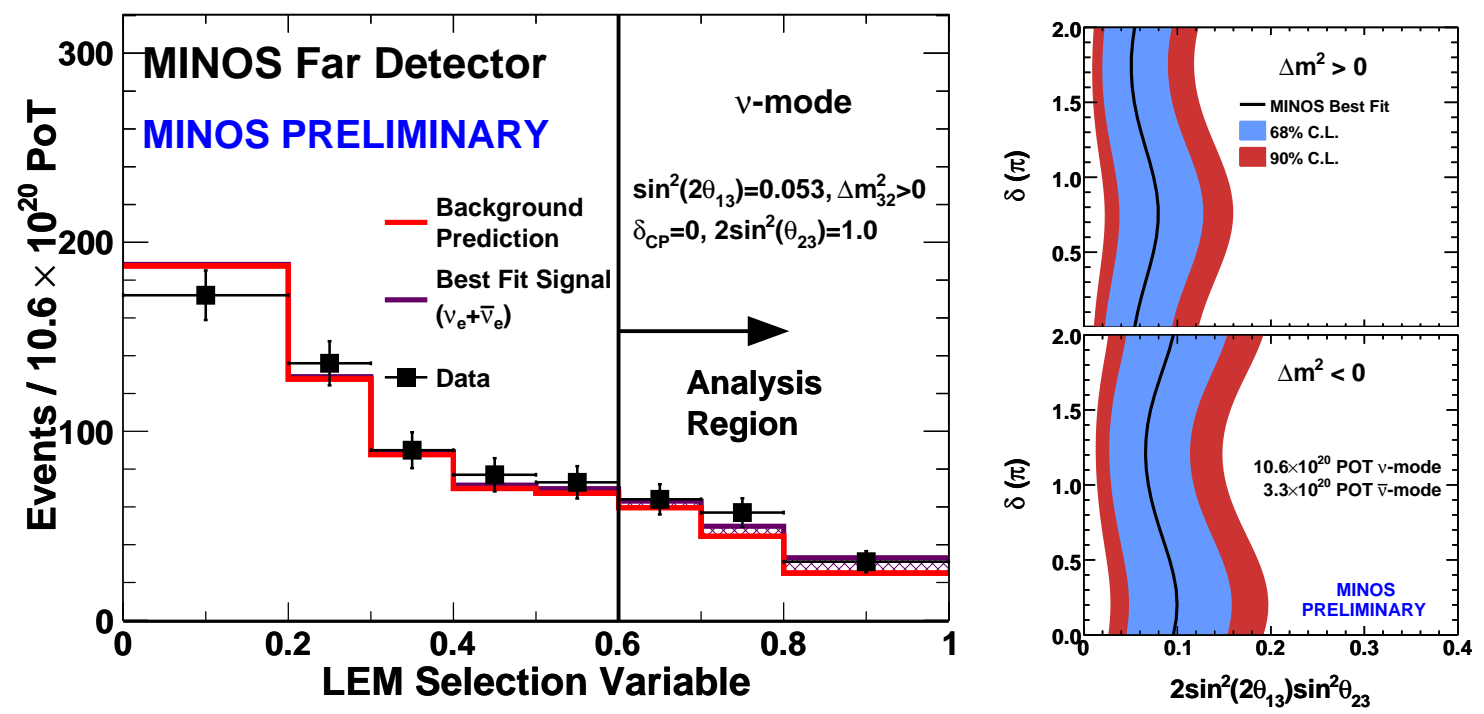

Figure 3: (left) LEM Particle ID for far detector events collected during $v_{\mu}$-dominated beam configuration. (right) The $68 \%$ and $90 \%$ confidence contours on $2 \sin ^{2}\left(2 \theta_{13}\right) \sin ^{2}\left(\theta_{23}\right)$ for the normal(top) and inverted(bottom) mass hierarchies.

and assuming that neutrinos and anti-neutrinos oscillate with the same parameters. In this combined analysis we have made the world's most precise measurement of the neutrino mass-splitting, $\left|\Delta m^{2}\right|=2.39_{-0.10}^{+0.09} \times 10^{-3} \mathrm{eV}^{2}$, and a competitive measurement of the mixing angle, $\sin ^{2}(2 \theta)=0.957_{-0.036}^{+0.035}$. If the data are fit separately, such that the parameters are not constrained to be the same, the difference between the anti-neutrino and neutrino mass splittings is measured to be $1.0_{-2.8}^{+2.4} \times 10^{-4} \mathrm{eV}^{2}$.

\section{3. $v_{e}$ Appearance Analysis}

The MINOS detectors were optimized for muon identification and the size of the scintillating strips makes the identification of electro-magnetic showers difficult (given their similarity to Neutral Current hadronic showers). A new technique, LEM (Library event Matching), was employed which compares each event to a large library of signal and background events. The event is classified as a signal or background event via a neural network with the reconstructed energy and three variables constructed from the 50 best-matches in the library as inputs [5]. The three main components of the background at the Near Detector $\left(\mathrm{NC}, v_{\mu} \mathrm{CC}\right.$ and intrinsic $\left.v_{e} \mathrm{~s}\right)$ are determined by fitting the charge current $v_{e^{-}}$ like event data sample in three different beam configurations (nominal, horn-off and high-energy mode) - each configuration has slightly different background components. These components are extrapolated separately to the far detector to obtain the predicted background to the $v_{e}$ appearance analysis. We expect to observe $128.6 \pm 32.5(17.5 \pm 3.7)$ events and observe 152 (20) events in the $v_{\mu}$-dominated $\left(\bar{v}_{\mu}\right.$-enhanced $)$ beam. Figure 3 plots the value of the LEM particle ID for Far Detector data collected in the $v_{\mu}$-dominated beam and the best-fits to $\theta_{13}$ using all the beam data. Assuming that neutrinos and anti-neutrinos oscillate the same we measure, at $\delta_{C P}=0,2 \sin ^{2}\left(2 \theta_{13}\right) \sin ^{2}\left(\theta_{23}\right)=0.053(0.094)$ for the normal(inverted) hierarchy.

\section{Conclusion}

We have presented measurements of the MINOS $v_{\mu} / \bar{v}_{\mu}$ disappearance and $v_{e}$ appearance analyses performed using the complete MINOS data sets. The complete MINOS data set includes $10.71 \times 10^{20}$ POTs collected in the $v_{\mu}$-dominated mode, $3.36 \times 10^{20}$ POTs in the $\bar{v}_{\mu}$-enhanced mode and a cumulative exposure to atmospheric neutrinos of 37.88 kton-yrs. Assuming that neutrinos and anti-neutrinos oscillate with the same parameters we measure $\sin ^{2}(2 \theta)=0.957_{-0.036}^{+0.035}$, $\left|\Delta m^{2}\right|=2.39_{-0.10}^{+0.09} \times 10^{-3} \mathrm{eV}^{2}$ and, for $\delta_{C P}=0$, constrain $2 \sin ^{2}\left(2 \theta_{13}\right) \sin ^{2}\left(\theta_{23}\right)$ to be between $0.01(0.03)$ and $0.12(0.19)$ at $90 \%$ C.L. for the Normal(Inverted) Hierarchy. If the latter fits are performed assuming the reactor best-fit for $\theta_{13}$ we have a small preference for the inverted hierarchy. 


\section{References}

[1] Michael, D.G et al. [MINOS Collaboration] Nucl. Instrum. Methods A 596 190-228 (2008)

[2] Crane, D.A. et al., [NuMI Beam Group] FERMILAB-TM-1946 (1995)

[3] Adamson, P. et al. [MINOS Collaboration] Phys. Rev. D 86 052007 (2012)

[4] Adamson, P. et al. [MINOS Collaboration] Phys. Rev. Lett. 108 191801 (2012)

[5] Adamson, P. et al. [MINOS Collaboration] Phys. Rev. Lett. 107 181802 (2011)

[6] Adamson, P. et al. [MINOS Collaboration] Phys. Rev. Lett. 106 181801 (2011)

[7] Barr, G. et al. Phys. Rev. D 74094009 (2006) 